testing using Abbott CT/NG. When unpooling the samples, one discordant result was solved. Three CT infections (1 in each samplingsite) and four NG infections (2A and $2 \mathrm{P}$ ) were missed; one CT (P) and three NG (A) infection were found to be false positive (one in each sampling site). This converts into a respective sensitivity and specificity of 91.2\% (95\% CI: 76.3-98.1\%) and 99.8\% (95\%CI: 99.0-100.0\%) for CT and 88.6\% (95\%CI: 73.3-96.8\%) and 99.4\% (95\%: 98.3-99.9\%) for NG of the pooling strategy. Cohen's Kappa agreement was 0.94 for CT and 0.89 for NG which is an almost perfect agreement.

Conclusion We showed that this pooling strategy performs well using the FDA approved point-of-care assay GeneXpert. This may be a very cost-effective strategy and also feasible, as the assay is widespread throughout the African continent for tuberculosis testing.

Disclosure No significant relationships.

\section{P542 PREVALENCE OF STIS AMONG MSM INITIATING PREP IN WEST-AFRICA (COHMSM-PREP ANRS 12369 - EXPERTISE FRANCE)}

${ }^{1}$ Irith De Baetselier*, ${ }^{2}$ Tania Crucitti, ${ }^{3}$ Issifou Yaya, ${ }^{4}$ Bintou Dembele, ${ }^{5}$ Ephrem Mensah, ${ }^{6}$ Elias Dah, ${ }^{7}$ Amadou Koné, ${ }^{8}$ Hortense Fayé-Ketté, ${ }^{9}$ Souba Diandé, ${ }^{8}$ Alain Yeo, ${ }^{10}$ Anoumou Dagnra, ${ }^{3}$ Christian Laurent, ${ }^{11}$ Bea Vuylsteke. ${ }^{1}$ Institute of Tropical Medicine, Clinical Sciences, Antwerp, Belgium; ${ }^{2}$ Institut Pasteur du Cameroun, Yaoundé, Cameroon; ${ }^{3}$ IRD, INSERM, Montpellier, France; ${ }^{4}$ ARCAD/SIDA, Bamako, Mali; ${ }^{5}$ Espoir Vie Togo, Lomé, Togo; ${ }^{6}$ Association African Solidarité, Ouagadougou, Burkina Faso; ' ${ }^{7} E R E F O / U C R C$, USTTB, Bamako, Mali; ${ }^{8} / n s t i t u t$ Pasteur Côte d'Ivoire, Abdijan, Côte d'Ivoire; ${ }^{9}$ LNR-TB, Ouagadougou, Burkina Faso; ${ }^{10} \mathrm{CHU}-\mathrm{SO}-\mathrm{LNR}$-TB, Lomé, Togo; ${ }^{11}$ Institute of Tropical Medicine, Department of Public Health, Antwerp, Belgium

\subsection{6/sextrans-2019-sti.617}

Background Men who have sex with men (MSM) coming forward for Pre-Exposure Prophylaxis (PrEP) are at high risk for HIV and other Sexually Transmitted Infections (STIs). However, little is known about the prevalence of STIs among MSM in West-Africa. Yet, understanding the STI epidemic among MSM will improve STI management. In the framework of a PrEP demonstration study in West-Africa (CohMSMPrEP), we tested all participants for STIs at enrollment.

Methods The study was conducted in Abidjan-Côte d'Ivoire, Bamako-Mali, Lomé-Togo and Ouagadougou-Burkina Faso. Participants $(n=507)$ were tested for the following STIs using the GeneXpert instrument: Chlamydia trachomatis (CT)/Neisseria gonorrhoeae (NG) in Anorectum (A), Urine (U) and Pharynx (P), and Trichomonas vaginalis (TV) in urine. Mycoplasma genitalium (MG) was tested using the S-DiagMGTV multiplex assay in A-U-P samples.

Results The overall prevalence of CT was $17.9 \% \quad(19.4 \%$, $22.0 \% 16.4 \%$, and $13.6 \%$ in Lomé, Abidjan, Bamako and Ouagadougou, respectively). Most CT infections were anorectal $(12.3 \%$, followed by urethral $(5.7 \%)$. In Bamako, the second most infected sample type was pharyngeal $(6.0 \%)$ instead of urine $(5.0 \%)$. Overall prevalence of $\mathrm{NG}$ was $15.8 \%(9.7 \%$; 25.0\%; 6.0\%, 22.3\% in Lomé, Abidjan, Bamako and Burkina, respectively). Most NG infections were found in the anorectum $(10.7 \%)$, followed by the pharynx (5.7\%). In Mali, no pharyngeal NG infections were detected. MG infection was $26.0 \%$ for Lomé and $27.6 \%$ for Ouagadougou (results for other sites not yet available). The majority of MG infections were found in the anorectum (15.4\%). Among all participants, only one urine sample with TV has been found in Bamako.

Conclusion We showed a very high prevalence of extra-genital STIs among PrEP users in West-Africa. We also detected infections which would not have been treated if a syndromic management approach would have been applied (87.9\%). In order to limit transmission of infections we recommend to test also extra-genital sites for STIs in this population.

Disclosure No significant relationships.

\section{P543 COST-EFFECTIVENESS OF PRE-EXPOSURE PROPHYLAXIS IN MSM WITH EVENT-DRIVEN AND DAILY REGIMENS}

${ }^{1}$ Maarten Reitsema*, ${ }^{1}$ Albert Jan Van Hoek, ${ }^{2}$ Maria Xiridou, 'Jacco Wallinga, ${ }^{2}$ Birgit Van Benthem, ${ }^{3}$ Ard Van Sighem, ${ }^{4}$ Maarten Schim Van Der Loeff, ${ }^{5}$ Maria Prins, ${ }^{4}$ Elske Hoornenborg. 'Centre for Infectious Diseases Control, National Institute for Public Health and The Environment, Bilthoven (RIVM), Bilthoven, Netherlands; ${ }^{2}$ National Institute for Public Health and the Environment (RIVM), Epidemiology and Surveillance, Centre for Infectious Diseases Control, Bilthoven, Netherlands; ${ }^{3}$ Stichting HIV Monitoring, Amsterdam, Netherlands; ${ }^{4}$ Public Health Service Amsterdam, Amsterdam University Medical Center (UMC), Infectious Diseases, Infection and Immunity (Aland II), Amsterdam, Netherlands; ${ }^{5}$ Public Health Service of Amsterdam, Amsterdam, Netherlands

\subsection{6/sextrans-2019-sti.618}

Background Pre-exposure prophylaxis (PrEP) is highly effective in reducing HIV transmission among men who have sex with men (MSM). We investigated the impact of daily and eventdriven PrEP on the transmission of HIV and N. gonorrhoeae (NG) and its cost-effectiveness in the Netherlands.

Methods We developed a stochastic agent-based transmission model of HIV and NG among MSM. We simulated three scenarios: (1) No PrEP; (2) Offering daily and event-driven PrEP; (3) Offering only daily PrEP. Three-monthly PrEP monitoring included testing for HIV, gonorrhoea, and other infections. From the Amsterdam PrEP Demonstration Project (AMPrEP) data, it was estimated that $27 \%$ of PrEP users prefer event-driven PrEP and they use half the amount of PrEP pills used by daily users. We assumed PrEP effectiveness was $86 \%$ regardless of regimen. Simulated outcomes of the transmission model were used in an economic model to calculate costs, quality-adjusted life-years (QALY), and incremental costeffectiveness ratios (ICER), over 2018-2027, taking a healthcare payer perspective. An ICER less than $€ 20,000$ per QALY gained was considered cost-effective.

Results PrEP resulted in 3,486 HIV infections averted and 1,482 QALYs gained over 2018-2027. Gonorrhoea prevalence dropped from $0.782 \%$ in 2017 to $0.023 \%$ in 2027. When offering both daily and event-driven PrEP, the costs for PrEP medication were $€ 19$ million over 2018-2027. This resulted in less total costs than when no PrEP is offered, making this programme cost-saving. With only daily PrEP, the costs for PrEP medication were $€ 22$ million over 2018-2027, making this programme cost-effective with a mean ICER of $€ 217.40$ per QALY gained.

Conclusion The PrEP programme (including STI monitoring) can be effective in reducing HIV incidence and gonorrhoea prevalence among MSM and can be cost-effective, even if all PrEP users prefer the daily regime. Monitoring of PrEP users can result in reductions in prevalence of STIs being monitored. Acknowledgements: AIDSfonds (2014037), ZonMw (522002003).

Disclosure No significant relationships. 